\title{
Study on Oilfield Distribution Network Reconfiguration with Distributed Generation
}

\author{
Fan Zhang ${ }^{\mathrm{a} *}$, Yuexi Zhang ${ }^{\mathrm{a}}$, Xiaoni Xin ${ }^{\mathrm{a}}$, Lu Zhang ${ }^{\mathrm{b}}$, Li Fan ${ }^{\mathrm{a}}$ \\ ${ }^{a}$ Changqing Water and Power Supply Department, Xi'an 710201, China \\ ${ }^{b}$ Changqing Meterial and Equipment Supply Department, Xi'an710018, China
}

\begin{abstract}
Distributed generation (DG) based on oilfield associated gas has the characteristics of dispersive and small capacity. According to load control type, DG is treated as PQ point in power flow calculation. A distribution network reconfiguration algorithm based on simulated annealing and immune is proposed in this paper. Boltzmann simulated annealing operator is used in selection operation, and method of immune vaccine is adopted also, both the diversity and convergence performance of population are improved. Based on the traits of infeasible solutions in evolution, a method of public switches matrix of loops is used to avoid the infeasible solutions and searching space is compressed effectively. The tested system shows the advantages of given method, and great benefits can be obtained in oilfield application.
\end{abstract}

Keywords: Distributed generation; distribution network reconfiguration; infeasible solution; simulated annealing; immune vaccine

\section{Introduction}

The reconfiguration has been a main means of optimization operation in distribution network power system. Reference [1] and [2] had given an interpretation: under the normal operating conditions, based on some basic constrains, the network structure can be reconfigured by changing the open/closed status of those sectionalizing and tie switches to get the objectives of reducing power loss, balancing loads, improving voltage profiles, and enhancing reliability of system. According to the existing researches about distribution network reconfiguration, the heuristic methods were difficult to find the global optimal solution, and intelligence methods had a deficiency that infeasible solutions could reduce the search efficiency. The infeasible solution problem was discussed in [3], but the operation proceeding based on binary code was complicated, and correction of infeasible proceeding was inefficient.

With the continuous development of new energy, the power supply mode which based on DG got widely used in small and medium-sized distribution network. The effects of DG in distribution network were given in some papers. Reference [4] and [5] analyzed the effects of power loss, power flow, voltage, and power quality of DG in distribution network. While the short-circuit current and direction of DG was discussed in [6], and basis of partition also was given. Reference [7] considered the load distribution in power flow calculation, and reference [8] studied the recovery reconfiguration in DG system. Reference [9] considered the DG as negative load, and used genetic algorithm in configuration, but the infeasible solution and low efficiency of solution researching were still exist.

In the distribution network with DG, the power flow calculation should be changed because of the transformation of the power supply. Based on the issue, a study on oilfield distribution network with DG is given in this paper, and a network reconfiguration method based on simulated annealing immune algorithm is proposed. The infeasible solutions are abandoned completely in evolutionary process. The reconfiguration results of typical example and actual power system show a good effect.

\footnotetext{
* Manuscript received June 15, 2012; revised August 8, 2012.

Corresponding author. Tel.: +86-29-86023572; E-mail address: swordsteen@126.com.
} 


\section{Characteristics of Oilfield Distribution Network with DG}

\subsection{Overview of the DG in Changqing oilfield}

Because of the characteristics of oilfield production and the existence of some objective factors, the planning and development of power system must follow with the expansion of production areas. Compared with the urban power system, the internal devices of oilfield power system are widely distributed, and the network lines are generally longer, the load points are extremely fragmented. Furthermore, the operation mainly focuses on the reliability of power supply, and the power loss rate is generally high.

In Changqing oilfield distribution network, because of the unique geographical features and rich crude oil associated gas resources, the network-connected generation units are fairly common. Therefore, how to reduce the power loss while make sure the reliability at the same time, is a primary issue for economic operation of oilfield power system. There are 119 associated gas power stations and 145 generator sets with a total installed capacity of nearly $50000 \mathrm{~kW}$ in Changqing oilfield at present. These power stations use micro turbine, mainly because of the small turbines using oil associated gas as the fuel have characteristics of small size, light weight, high efficiency, less pollution, simple operation and maintenance. On the other hand, the DG also brings some problems to the power load distribution, power rate adjustment, and accident disposal, which makes a certain influence on the safe and optimal operation of the oilfield power system.

\subsection{Disposal of $D G$ node}

Done the connection with the power system, the system structure with DG turns from the distributed network to the network which supply and user side are associated. Reference [10] proposed that the selection of PQ, PV and balance nodes of DG should be considered integratively because of the power flow calculation model of DG is not same with the traditional generator set.

Although the incorporation of DG to the system can change the voltage of system node, reference [5] pointed out that the small and medium-capacity DG involved in the voltage regulator few directly. Then the DG can be seen as a controllable load at the system level. Because of the exploitation of associated gas and crude oil production keep a well pace, the generator output and load power is relatively stable compared with wind solar and other DG systems. The DG based on gas turbine can be simplified with a constant power model and the DG nodes can be seen as negative load points in power flow calculation.

\section{Reconfiguration Algorithm of Oilfield Distribution Network with DG}

\subsection{Mathematic model of distribution network reconfiguration}

The loss minimum formulation for reconfiguration problem, generally, can be mathematically expressed as follows:

$$
\min P_{\text {loss }}=\sum_{i=1}^{N_{b}} r_{i} \frac{P_{i}^{2}+Q_{i}^{2}}{V_{i}^{2}}, \text { subject to: } g(x)=0, S_{i} \leqslant S_{i, \max }, V_{i, \min } \leqslant V_{i} \leqslant V_{i, \max }
$$

where $P_{\text {loss }}$ is the power loss of system; $N_{b}$ is total number of branches; $r_{i}$ is resistance of branch $i ; P_{i}, Q_{i}$ represents active and reactive power of branch $i$ respectively; $V_{i}$ is voltage of the head node of branch $i$; $g(x)$ represents topology constraints, which ensure radial structure of each candidate topology; $S_{i, \max }$ is the maximal capacity of branch $i$; $V_{i, \min }, V_{i, \max }$ is voltage boundary of branch $i$.

\subsection{Encoding}

Consider to the characteristic of closed-loop design and open-loop operation of distribution network, the decimal encoding method based on loops is adopted in this paper, and chromosome size is obtained from the number of tie switches. Meanwhile, a taboo rule is also given: the switches that exist in none loops should be closed, otherwise a supply island could be formed. 


\subsection{Improved disposal of solution}

In [11], the problem of infeasible solutions was discussed in detail, but the identification method proposed was miscellaneous. An improved disposing method of solutions is used here to make sure the solutions are feasible, its main idea is storing public switches in advance, and the matrix of public switches between loops can be expressed as follows:

$$
\mathbf{S}=\left[\begin{array}{cccc}
1 & \vec{S}_{12} & \ldots & \vec{S}_{1 l} \\
0 & 1 & \ldots & \vec{S}_{2 l} \\
\vdots & \vdots & \vdots & \vdots \\
0 & 0 & \ldots & 1
\end{array}\right]
$$

where $\mathrm{S}$ is an upper triangular matrix, $\vec{S}_{12}=\left\{k_{1}, k_{2} \cdots\right\}, \vec{S}_{12}$ shows the public switches between loop 1 an loop 2.



Fig. 1. IEEE 33-bus system

The elaborate disposing method of individual can be explained as follows: Start from the first gene of the chromosome individual, choose switch $k_{1}$ which belongs to loop 1 and make it open. Then choose the switch $k_{i}^{\prime}$ of loop 2, if both $k_{1}$ and ${k_{i}^{\prime}}_{i}$ are the public switches between loop 1 and loop 2, it means that the public switches are opened twice. And then the switch of gene 2 should chosen in the range of switches expect the public switches between loop 1 and loop 2. The same strategy can be adopted on the other genes of the individual.The $\mathbf{S}$ of IEEE 33-bus system which expressed in Fig. 1 can be showed as follows:

$$
\mathbf{S}=\left[\begin{array}{ccccc}
1 & 0 & \vec{S}_{13} & \vec{S}_{14} & \vec{S}_{15} \\
0 & 1 & \vec{S}_{23} & \vec{S}_{24} & 0 \\
0 & 0 & 1 & \vec{S}_{34} & 0 \\
0 & 0 & 0 & 1 & \vec{S}_{45} \\
0 & 0 & 0 & 0 & 1
\end{array}\right]
$$

where $\vec{S}_{13}=\{33\}, \vec{S}_{14}=\{6,7\}, \vec{S}_{15}=\{3,4,5\}, \vec{S}_{23}=\{9,10,11\}, \vec{S}_{24}=\{34\}, \vec{S}_{34}=\{8\}, \vec{S}_{45}=\{25,26,27,28\}$.

The creation of individuals can be expressed as follows: 1) Select the switch 4 randomly in the switch group $(2,3,4,5,6,7,33,21,20,19,18)$ of loop 1.2) Select the switch 10 randomly in the switch group $(9,10,11,12,13,14,34)$ of loop 2 , the taboo rule does not implied here because $\vec{S}_{12}$ is empty. 3) As to the loop 3, search the third column. Because it has public switches with loop 1 and 2, then search the switch 8 randomly while exclude the choices of switch 9, 10, and 11. 4) Search the forth column, loop 4 has public switches with loop 1, 2 and 3. Then taboo out the choice of switch 8 and select switch 28 randomly from switch group $(6,7,8,34,15,16,17,36,32,31,30,29,28,27,26,25)$ of loop 4. 5) Loop 5 has public switches with loop 1 and 4, and the switch 4 of loop 1 which exists in $\vec{S}_{15}$ has been selected, while switch 28 of loop 4 which exists in $\vec{S}_{45}$ has been selected. So the selection of switch in loop 5 should exclude the same switch of $\vec{S}_{15}$ and $\vec{S}_{45}$, then choose to open switch 24 randomly. 6) After the operation, a feasible solution can be obtained, and the open switch group is $(4,10,8,28,24)$. 
Through the discrimination method, all the solutions will be feasible. Meanwhile, in the evolution of population, the method is useful while gene of individual changes.

\section{Optimization Based on Simulated Annealing Immune Algorithm}

\subsection{Selection}

The method of selection is an important process of evolution all the time. The common method such as roulette selection, while just based on the fitness of individual, usually results in local optimum. In this paper, Boltzmann simulated annealing operator is used to select new solutions. Its advantage is that some inferior solutions can also be chosen to join into evolution, and diversity of population is remained preferably. According to reference [12], initial temperature can be confirmed by the relative performance between fitness function and original population. The expression of initial temperature can be showed as follows:

$$
t_{0}=\frac{\left(f_{i \min }-f_{j \max }\right)}{\ln \left(f_{i \min } / f_{j \max }\right)}
$$

where $f_{\text {imin }}, f_{\text {jmax }}$ represents the minimal and maximal fitness in initial population respectively. The initial temperature considers relative performance of population and bad infection of evolution is avoided promptly. And annealing temperature can be showed by (6):

$$
t_{k+1}=\alpha t_{k}, \quad k \geq 0, \quad 0<\alpha<1
$$

where $\alpha$ is a proportion factor, and a large $\alpha$ means that temperature descends much slowly. After the determination of temperature, Boltzmann annealing operator in [13] can be used to calculate the select probability, which has the expression in (7).

$$
p_{i}=\frac{\exp \left\{-\frac{f(i)-\bar{f}}{t_{k}}\right\}}{\sum_{j=1}^{N} \exp \left\{-\frac{f(i)-\bar{f}}{t_{k}}\right\}}
$$

where $\bar{f}$ is the average fitness of the population, according to (7), the select probability of individuals relates the integral performance of the population. Then some bad solutions can be taken to next population and get evolution, so local optimum is avoided effectively.

\subsection{Vaccine and inoculation}

The vaccine of immune algorithm is an estimation of optimal individual gene which obtained by the prior knowledge of evolution. Reference [11] points that the distribution network reconfiguration differs from other problems, such as Travelling Salesman Problem (TSP), its prior knowledge is difficult to acquire. In [11], the vaccine extraction method does not consider all genes, because some information of global solution still exists in the different genes between the best individuals. In this paper, a refined vaccine extraction method is proposed. A matrix $\mathbf{B}$ is defined, its size is $2 \times l$, while $l$ is the size of individual. Matrix B is composed by historic and present optimal individuals, which expressed by (8).

$$
\mathbf{B}=\left[\begin{array}{ccccc}
H_{1} & H_{2} & \cdots & H_{l-1} & H_{l} \\
P_{1} & P_{2} & \cdots & P_{l-1} & P_{l}
\end{array}\right]
$$

After the operation of simulated annealing, choose some individuals of the population, compare with inoculation probability $V_{p}$ and the random number $\lambda$ which created by random function. If $\lambda>V_{p}$, the inoculation operation is executed, otherwise, inoculation is avoided. The idea of inoculation is that choose 
a column $j$ of $\mathbf{B}$, then compared the gene $j$ of $H_{j}$ with $P_{j}$, if get a same result, the inoculation is canceled, otherwise replace the gene in same order with gene $j$. After the inoculation, calculate the fitness of new individual. If the fitness descends, it indicates that inoculation is degenerate and should be canceled. Otherwise, inoculation is effective and the new individual gets the permission to next generation.

\subsection{Hyper-mutation and immune supplement}

In the population, find the individual $i$ which has a middle fitness. Then compared with individual $j$, choose one gene of individual $j$ and execute the mutation operation if the value of $f(i)$ less than or equal to $f(j)$, otherwise choose two genes and execute mutation operation. The variant individuals and forgoing population compose a new population which has a number of $2 N$. Along with the evolution, abound metabolisms exist in immune cells. On the foundation of alternation of cells, new individuals which created randomly are used to replace the individuals with bad performance. The amount of replacing is according to the size of population. Through the supplement, the diversity can be kept in evolution.

\section{Test results}

In this paper, the proposed algorithm is tested on IEEE 33 bus system which given in [9], the parameters of DG is showed in Table 1.

Table.1. Data of DG in reference [9]

\begin{tabular}{lll}
\hline Num & Installation node & Active and reactive power \\
\hline 1 & 4 & $-51-\mathrm{j} 31.61$ \\
2 & 8 & $-90-\mathrm{j} 43.59$ \\
3 & 25 & $-180-\mathrm{j} 87.18$ \\
4 & 30 & $-200-\mathrm{j} 150$ \\
\hline
\end{tabular}

The size of chromosome is set as 5 based on the encoding method of previous segments in this paper, and some parameters are chosen as follows: size of population equals 30, maximum generations is given the number of $60 . \alpha$ and the number of vaccine supplement is assigned value of $0.9,5$ respectively. The vaccine inoculation number is set as 6 . In order to compare the effectiveness of the proposed algorithm, the genetic algorithm with infeasible solution disposal is tested in the example, and the crossover rate and mutation rate of genetic operation is set as $0.9,0.2$ respectively. The comparison of random results showed in Fig. 2.

Both algorithms can obtain the optimal solution, the opened switches group of optimal reconfiguration structure is $(7,14,9,32,28)$, and the minimal power loss is $96.56 \mathrm{~kW}$. The optimal network result is same as the results in [9], and the power loss difference is caused by the flow calculation.

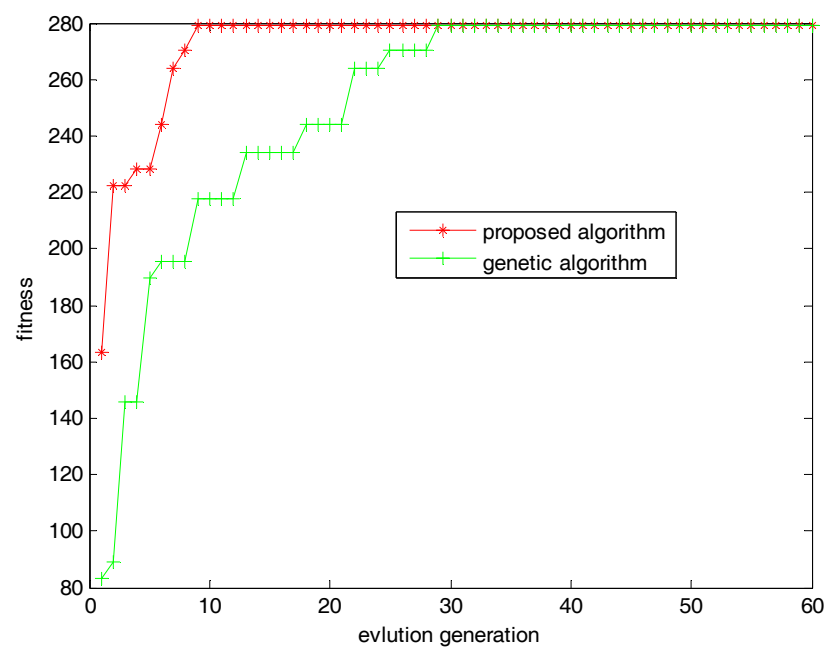

Fig. 2. Results of genetic algorithm and proposed algorithm 
Fig. 2 shows that the evolution generation is 9 to get the optimal result by using proposed algorithm while genetic algorithm needs 29 . The searching speed of proposed method is much faster than the genetic algorithm because of the selection operator can avoid the singleness of evolutionary population, and the vaccine inoculation, mutation, and supplement can reserve the best gene of individuals, therefore the global optimal result can be searched quickly.

The practical calculation example is a $10 \mathrm{kV}$ system of Xinhe substation in Ansai oilfield of Changqing. The system includes 8 distribution lines with overall load power of $8000 \mathrm{~kW}$. The simplified structure is showed in Fig. 3, there are 29 load points, 28 line switches, 5 loop switches and 7 DG sets. Table 2 gives the data of load points and Table 3 shows the data of DG.

The rated voltage of system is $10 \mathrm{kV}$ and power factor of generator is 0.75 . The operator parameters of the proposed algorithm are same with the test example, the reconfiguration result showed in Table 4.

Compared with the reconfiguration without DG, the power loss of reconfiguration with DG can get a significantly reduction, but the voltage quality gets a slight decrease because of the non-optimal installation location and capacity of DG, and the lowest node voltage drops from 0.9267 to 0.9257 . Reference [5] discussed the voltage support of DG in detail, so it does not elaborated in this paper. Furthermore, the DG which studied in this paper is assumed that has been built and the planning of DG is not considered.



Fig. 3. $10 \mathrm{kV}$ diagram of Xinghe substation

Table. 2 Active and reactive power of load nodes

\begin{tabular}{cll|lll|lll|lll}
\hline Node & $\mathrm{P}(\mathrm{kW})$ & $\mathrm{Q}(\mathrm{kvar})$ & Node & $\mathrm{P}(\mathrm{kW})$ & $\mathrm{Q}(\mathrm{kvar})$ & Node & $\mathrm{P}(\mathrm{kW})$ & $\mathrm{Q}(\mathrm{kvar})$ & Node & $\mathrm{P}(\mathrm{kW})$ & $\mathrm{Q}(\mathrm{kvar})$ \\
\hline $1-3$ & 0 & 0 & 10 & 559 & 347 & 17 & 503 & 312 & 24 & 417 & 258 \\
4 & 672 & 417 & 11 & 703 & 436 & 18 & 222 & 137 & 25 & 0 & 0 \\
5 & 159 & 99 & 12 & 163 & 101 & 19 & 0 & 0 & 26 & 65 & 40 \\
6 & 459 & 285 & 13 & 1518 & 941 & 20 & 64 & 40 & 27 & 50 & 31 \\
7 & 842 & 522 & 14 & 50 & 31 & 21 & 264 & 163 & 28 & 213 & 132 \\
8 & 0 & 0 & 15 & 506 & 313 & 22 & 19 & 12 & 29 & 286 & 177 \\
9 & 199 & 124 & 16 & 62 & 39 & 23 & 64 & 40 & & & \\
\hline
\end{tabular}

Table.3. Data of DG in Xinghe $10 \mathrm{kV}$ system

\begin{tabular}{ccc}
\hline Num & Installation node & installed capacity $(\mathrm{kW})$ \\
\hline 1 & 4 & 800 \\
2 & 13 & 900 \\
3 & 15 & 400 \\
4 & 16 & 200 \\
5 & 19 & 125 \\
6 & 22 & 100 \\
7 & 23 & 300 \\
\hline
\end{tabular}


Table.4. Reconfiguration result of Xinghe $10 \mathrm{kV}$ system

\begin{tabular}{llll}
\hline Items & Pre. & Without DG & With DG \\
\hline & 29 & 29 & 29 \\
Tie switches set & 30 & 12 & 12 \\
& 31 & 31 & 18 \\
Losses(kW) & 32 & 15 & 15 \\
Lowest voltage & 53 & 27 & 27 \\
\hline
\end{tabular}

\section{Conclusions}

In this paper, a reconfiguration algorithm which considers the characteristics of DG in oilfield distribution network is proposed. The characteristic of associated gas is analyzed, and DG points are treated as PQ nodes in power flow calculation. The disposal of infeasible solution is studied in detail, on the basis of decimal encoding, the upper triangular matrix of public switches between loops is created and all the solutions are feasible, and the evolution efficiency is improved. The combination of simulated annealing operator and immune operator accelerates the searching speed of global optimization and obtains a better performance of population. The practical example shows that the reconfiguration with DG has great economic prospects in oilfield optimization.

\section{References}

[1] Liu L, Chen X. Reconfiguration of distribution networks based on fuzzy genetic algorithm. Proceedings of the CSEE, 2000; 20(2):66-69.

[2] Liu W, Han Z. Distribution network reconfiguration based on optimal flow pattern algorithm and genetic algorithm. Power System Technology, 2004; 28(19): 29-33.

[3] Huang Y, Li X. Study on infeasible solution of distribution network reconfiguration genetic algorithm. Electric Power Construction, 2004; 25(3):23-27.

[4] Li B, Li X. Distribution generation sources and their effects on distribution networks. International Electric Power for China, 2005; 9(3):45-49.

[5] Wang Z, Zhu S, Zhou S, et al. Impacts of distributed generation on distribution system voltage profile. Automation of Electric Power System, 2004; 28(16):56-60.

[6] Wen Y, Wang X. Effect of distributed generation on protection in distribution system. Relay, 2008; 36(1):12-14.

[7] Yang X, Duan J, Yang W. Power flow calculation based on power losses sensitivity for distribution system with distributed generation. Power System Technology, 2009; 33(18):139-143.

[8] Lu Z, Dong Y. Service restoration strategy for the distribution system with DGs. Automation of Electric Power System, 2007; 31(1):89-92.

[9] Cui J, Liu T, Li X. Network reconfiguration at the distribution system with distributed generation. Power System Protection and Control, 2008; 36(15):37-40.

[10] Wang S, Huang L, Wang C, Li D. Unbalanced three-phase power flow calculation for distributed power generation, Electric Power Automation Equipment, 2007; 27(8):11-15.

[11] Yu J, Zhang F. Distribution network reconfiguration based on refined immune genetic algorithm. Power Systems Technology, 2009; 33(19):100-105.

[12] Wang L, Zheng D. A class of improved evolutionary programming and its optimization performances analysis. Computer Engineering and Applications, 2002; 38(1):8-10.

[13] Li Y, Yao J, Yao D. An efficient composite simulated annealing algorithm for global optimization. In Proc. of IEEE 2002 International Conference on Communications, Circuits and Systems and West Sino Expositions, 2002:1165-1169. 